\title{
Evaluation of response to protease inhibitor based first line anti-retroviral therapy (ART) regimens in human immunodeficiency virus infected children compared to standard first line non-protease inhibitor ART regimens: an Indian experience
}

\author{
*Sowmya Shashidhara', Sangita Trivedi ${ }^{2}$, Jayesh Kosambiya ${ }^{2}$, Aditya Gaur ${ }^{3}$ \\ Sri Lanka Journal of Child Health, 2017; 46(2): 160-164
}

\begin{abstract}
Background: Children exposed to single dose nevirapine at birth, if tested human immunodeficiency virus (HIV) positive, cannot be given nevirapine based anti-retroviral therapy (ART). Therefore protease inhibitor (PI) based combinations are being used as first line therapy in such children. However, experience is limited regarding their efficacy and adverse effects in the Indian population.
\end{abstract}

Objectives: To document clinical and immunological responses to PI based regimens as first line therapy in HIV infected children less than 5 years old compared to responses to standard nonprotease inhibitor (NPI) first line regimens recommended by National AIDS Control Organisation and World Health Organisation.

Method: An observational prospective cohort study was conducted in a tertiary care hospital in Surat, India, from December 2011 to June 2013 on children less than 5 years of age with confirmed HIV status. At diagnosis, clinical staging, growth parameters and CD4 levels were assessed and the children were allotted to NPI and PI groups depending on prior nevirapine exposure. Clinical, immunological and biochemical alterations after ART initiation were assessed and compared after 6 months of therapy.

Results: During the study period, there were 43 children less than 5 years of age with confirmed HIV status. Children in both NPI and PI groups showed significant improvement in clinical stage of the disease after 6 months of therapy $(\mathrm{P}<0.001)$.

${ }^{1}$ Kasturba Medical College, India, ${ }^{2}$ Government Medical College, Surat, India, ${ }^{3}$ St. Jude Research Centre, Memphis, USA

*Correspondence:sowmyashashidhara86@gmail.com (Received on 30 June 2016: Accepted after revision on 10 October 2016)

The authors declare that there are no conflicts of interest

Personal funding was used for the project.

Open Access Article published under the Creative Commons Attribution CC-BY CC
Fewer children remained in stages 3 and 4 at the end of the study in the PI group. The mean height, weight and CD4 counts of children in the PI group were significantly higher than those in NPI group after 6 months of ART therapy. Total white blood cell counts and cholesterol levels were considerably higher in the PI group $(\mathrm{p}<0.001)$.

Conclusions: PI based regimens were effective as first line therapy in HIV infected children less than 5 years old. Both PI and NPI regimens were equally effective at 6 months of therapy but PI based regimens achieved significantly better growth parameters and CD4 levels. Elevated total cholesterol levels were observed after therapy with PI regimens.

DOI: http://dx.doi.org/10.4038/sljch.v46i2.8274

(Keywords: HIV, children, protease inhibitors)

\section{Introduction}

In India, first line antiretroviral therapy (ART) regimens extensively used for children and adults with human immunodeficiency virus (HIV) comprise 3 drugs viz. stavudine or zidovudine, lamivudine and nevirapine ${ }^{1,2,3}$. Children given single dose nevirapine at birth for prevention of parent to child transmission cannot receive nevirapine based ART regimens according to the National AIDS Control Organisation (NACO) policy $^{3,4}$ and are prescribed protease inhibitor (PI) based combinations containing lopinavir / ritonavir $^{5,6}$. Experience is limited regarding clinical and immunological responses of PI regimens as first line therapy in children in the Indian population. Hence this study was undertaken.

\section{Objectives}

To document clinical and immunological responses to PI based regimens as first line therapy in HIV infected children less than 5 years old compared to responses to standard non-protease inhibitor (NPI) first line regimens recommended by National AIDS Control Organisation and World Health Organisation.

\section{Method}

An observational prospective cohort study was conducted from December 2011 to June 2013 in a 
tertiary care hospital in a high HIV burden region of Western India. All children aged less than 5 years with confirmed HIV infection, ART naïve except for exposure to nevirapine at birth and eligible for first line ART were included in the study $^{3}$. The study was conducted with approval from the institutional ethics review committee.

HIV infection was confirmed by deoxyribonucleic acid polymerase chain reaction (DNA PCR) in children less than 18 months and the enzyme linked immunosorbent assay (ELISA) in older children.
Clinical staging was done according to WHO staging system with relevant history taking and physical examination, including anthropometry ${ }^{5,6}$. Investigations were as recommended by NACO, including specific tests for diagnosis of tuberculosis (TB) and other comorbidities. Children eligible for ART after clinical and immunological staging were started on ART and allocated to NPI and PI groups as shown in Table 1. Routine care like immunization, cotrimoxazole prophylaxis, feeding/diet was advised to all children with HIV infection ${ }^{5,6}$.

Table 1: Division of study groups based on ART drug regimens

\begin{tabular}{|l|l|}
\hline \multicolumn{1}{|c|}{$\begin{array}{c}\text { Standard first line therapy } \\
\text { Non-protease inhibitor (NPI) group }(n=31)\end{array}$} & \multicolumn{1}{c|}{$\begin{array}{c}\text { Alternate first line therapy } \\
\text { Protease inhibitor (PI) group }(n=12)\end{array}$} \\
\hline 1. Zidovudine-lamivudine-nevirapine & 1. Zidovudine- lamivudine- lopinavir/ritonavir \\
2. Stavudine-lamivudine-nevirapine & 2. Stavudine - lamivudine- lopinavir/ritonavir \\
3. Zidovudine-lamivudine-efavirenz (if co- & \\
$\quad \begin{array}{l}\text { infection with TB was present) } \\
\text { 4. Stavudine-lamivudine-efavirenz (if co- } \\
\text { infection with TB was present) }\end{array}$ & \\
\hline
\end{tabular}

All children included in the study were followed up monthly at the ART centre, Surat and at each visit, history of clinical events, anthropometrical measurements and examination were done by the same physician using the same instruments to determine clinical staging. If illnesses were present, their type, course and frequency were assessed. Severity was assessed by the need for in-patient admission. There were no drop-outs. Adherence, compliance and preference for drug intake and occurrence of adverse effects were evaluated. All biochemical parameters determined on enrollment, were repeated 6 monthly or if the child developed any clinical events. Immunological status was monitored 6 monthly by repeating CD 4 counts. The baseline staging and the final outcome at the end of 6 months of initiating ART were compared by the Chi square test. Comparison of the weight and height patterns and the mean CD4 counts were done using the t-test.
The end points of the study were improvement of clinical stage and increase in the CD4 counts after 6 months of initiating ART therapy. Even though there were many infants exposed to nevirapine at birth, only those infants with confirmed infection and eligible for ART according to clinical and immunological criteria, were included. Those who were exposed, who were DNA PCR positive for HIV but did not fit the criteria for ART were not included as they did not qualify for ART. At the time of this study, guidelines to start alternate first line regimens were relatively new, so the group to whom PI was given is small. As this is an observational study, equal numbers of children could not be allocated to each group.

\section{Results}

Forty three children met the study eligibility criteria. Of them, 12 belonged to the PI group, and 31 to the NPI group. Baseline characteristics of study group are shown in table 1.

Table 2: Baseline characteristics of PI and NPI groups

\begin{tabular}{|l|c|c|}
\hline \multicolumn{1}{|c|}{ Variable } & Protease inhibitor group $(n=12)$ & Non-protease inhibitor group $(n=31)$ \\
\hline Mean age at diagnosis (years) & 0.7 & 2.4 \\
\hline Mean age at ART initiation (years) & 1.1 & 3.2 \\
\hline Sex distribution & 08 & 22 \\
Male & 04 & 09 \\
Female & & 22 \\
\hline Mode of delivery & 12 & 09 \\
Hospital delivered & 0 & positive \\
Home delivered & positive & \multicolumn{2}{|c|}{} \\
\hline Maternal HIV status & & \\
\hline
\end{tabular}

Baseline clinical staging of both groups showed that all 12 enrolled children in the PI group were in stages 1-3 with none in stage 4 (Figure 1). The 31 enrolled children in the NPI group were in all 4 stages with the largest number in stage 2 and the least in stage 4 (Figure 1). 

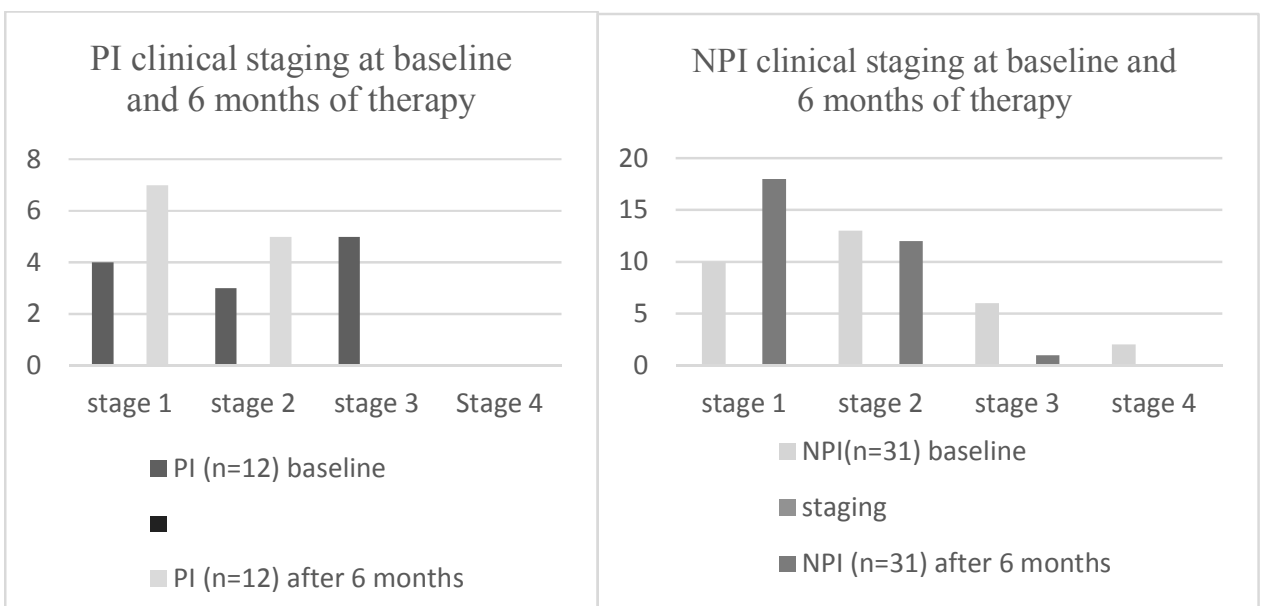

Figure 1: Comparing clinical staging of PI and NPI groups at baseline and after 6 months of ART initiation

After 6 months of ART initiation in the PI group, 7 $(58.3 \%)$ children were in stage one, $5(41.7 \%)$ in stage two and none in stages three or four. In the NPI group, $18(58.1 \%)$ children were in stage one, $12(38.7 \%)$ in stage two and $1(3.2 \%)$ in stage 3 (Figure 1). With PI regimens, $8(67.1 \%)$ were symptomatic at the beginning of ART therapy and $5(41.6 \%)$ remained symptomatic after 6 months.
With NPI regimens $21(67.8 \%)$ were symptomatic at the beginning of ART therapy and 13 (41.9\%) remained symptomatic after 6 months. On applying the Chi square test, both groups showed significant improvement at the end of 6 months $(\mathrm{p}<0.001)$. Comparison of clinical staging using Chi square test of both groups after 6 months of therapy did not show a significant difference $(\mathrm{p}=0.23)$.

Table 3: Comparison of PI and NPI regimens at 6 months after ART initiation

\begin{tabular}{|l|c|c|c|}
\hline \multicolumn{1}{|c|}{ Characteristic } & $\begin{array}{c}\text { PI Group }(\mathbf{n}=12) \\
\text { Mean } \pm \text { SD }\end{array}$ & $\begin{array}{c}\text { NPI Group (n=31) } \\
\text { Mean } \pm \text { SD }\end{array}$ & p value \\
\hline Weight $(\mathrm{kg})$ & $8.992 \pm 2.7681$ & $14.403 \pm 5.3816$ & $\mathrm{P}=0.002$ \\
\hline Height $(\mathrm{cm})$ & $70.92 \pm 8.447$ & $82.87 \pm 8.628$ & $\mathrm{P}=0.0002$ \\
\hline CD4 levels $(\mathrm{cell} / \mathrm{\mu l})$ & $1934 \pm 604.996$ & $693.71 \pm 469.32$ & $\mathrm{P}=0.0001$ \\
\hline Haemoglobin $(\mathrm{g} / \mathrm{dl})$ & $9.883 \pm 2.08$ & $10.532 \pm 1.3639$ & $\mathrm{P}=0.2362$ \\
\hline Total white cell counts $(\mathrm{x} \mathrm{1000/cu} \mathrm{mm)}$ & $9.858 \pm 2.05$ & $7.403 \pm 2.0474$ & $\mathrm{P}=0.0011$ \\
\hline Platelet counts $(\mathrm{x}$ 1000/cu mm) & $260.92 \pm 70.8$ & $311.77 \pm 111.515$ & $\mathrm{P}=0.1510$ \\
\hline Mean corpuscular volume (fl/cell) & $70.5 \pm 10.25$ & $77.62 \pm 11.344$ & $\mathrm{P}=0.0654$ \\
\hline Creatinine $(\mathrm{mg} / \mathrm{dl})$ & $0.625 \pm 0.135$ & $0.626 \pm 0.139$ & $\mathrm{P}=0.9831$ \\
\hline Total bilirubin levels $(\mathrm{mg} / \mathrm{dl})$ & $0.283 \pm 0.203$ & $0.284 \pm 0.1344$ & $\mathrm{P}=0.9850$ \\
\hline Alanine transaminase $(\mathrm{IU} / \mathrm{l})$ & $47.83 \pm 60.73$ & $30.19 \pm 23.913$ & $\mathrm{P}=0.1742$ \\
\hline Random blood sugar $(\mathrm{mg} / \mathrm{dl})$ & $86.5 \pm 8.68$ & $91 \pm 7.69$ & $\mathrm{P}=0.1043$ \\
\hline Amylase $(\mathrm{IU} / \mathrm{l})$ & $30.75 \pm 16.018$ & $26.81 \pm 15.087$ & $\mathrm{P}=0.4544$ \\
\hline Total cholesterol $(\mathrm{mg} / \mathrm{dl})$ & $155.92 \pm 35.357$ & $60.52 \pm 12.495$ & $\mathrm{P}=0.0001$ \\
\hline
\end{tabular}

The mean weight of children in PI group after 6 months of therapy was significantly different from that of NPI group $(p<0.01)$ and the mean height of children in PI group after 6 months of therapy was significantly different from that of NPI group $\mathrm{p}<0.001$ ) as shown in Table 3. The average percentage weight gains in the PI and NPI groups were $23.4 \%$ and $14.2 \%$ respectively whilst the average percentage height gains in the PI and NPI groups were $5.1 \%$ and $2.4 \%$ respectively after 6 months of ART initiation. The mean CD4 levels of children in PI group after 6 months of therapy was significantly different from that of the NPI group $(p<0.001)$ and the mean total white blood cell count of children in PI group after 6 months of therapy was significantly different from that of NPI group, $(\mathrm{p}<0.01)$ as shown in Table 3. The average percentage increase in CD4 in the NPI and PI groups was $66.3 \%$ and $75.3 \%$ respectively after 6 months of ART initiation. The mean total cholesterol of children in PI group after 6 months of therapy was significantly different from that of NPI group $(p<0.001)$ as shown in Table 3. The means of haemoglobin, platelet count, corpuscular volume, serum creatinine, total bilirubin, alanine transaminase, serum amylase and blood sugar in the PI group and NPI groups did not show any significant differences (Table 3). 


\section{Discussion}

The introduction of alternate first line regimens has given the opportunity for many infants with perinatal HIV infection to survive and thrive. However, studies evaluating their response in resource limited settings are few. The comparison of the impact on the clinical staging of both PI and NPI groups showed that both drug regimens were equally effective in improving the clinical stage of HIV disease, but fewer children remained in stages 3 and 4 at the end of the study in the PI group. The children on PI regimens showed a significant difference in anthropological parameters like height and weight $(\mathrm{p}<0.001)$. Also, the immunological outcome showed a higher mean CD4 count levels along with a significant difference in mean CD4 counts after 6 months of ART initiation and the average percentage increase in CD $4 \mathrm{~s}$ was $10 \%$ more in the PI group. These observations from our study suggest that children if treated with PI regimen, remain asymptomatic, achieve better growth parameters and have greater immune recovery.

In a study done by Violari et all comparing the clinical outcome of nevirapine versus ritonavirboosted lopinavir regimens in 288 children, superior outcome was seen with better CD4 counts in the PI group and higher treatment failure rates in the nevirapine group ${ }^{6}$. The Penpact study done on 266 children compared immunological outcome after initiation of NNRTI/NRTI versus PI regimens and showed that good long-term outcomes were achieved with all treatments strategies ${ }^{7}$. Jaspan HB et al, compared the two year outcomes of children on NNRTI/PI regimens in a South African paediatric antiretroviral program in 391 children in South Africa and achieved a sustained doubling of median CD4\% from baseline, and survival of $91 \%$, at 24 months $^{8}$. Contri et al, evaluated the nutritional status of HIV positive children and adolescents using ART in 23 children and adolescents on PI based regimens in comparison with 36 on NPI regimens and concluded that there was no significant difference in the nutritional status and anthropometric parameters ${ }^{9}$.

The most common alteration in the biochemical profile in this study was increase in the total serum cholesterol (16.6\%) in the PI group. Nausea and vomiting were the most common adverse effects observed but the PI group had a comparatively lesser incidence $(9.6 \%)$ than the NPI group $(16.5 \%)$. Other severe adverse effects were not reported during the study period. Contri et al evaluated the response of 23 children receiving PI based regimens and found that serum triglyceride levels and non-HDL-cholesterol levels were significantly higher in the PI group compared with the non-PI group, at any time point ${ }^{9}$. Charakida et al evaluated the lipid profile in 31 children receiving PI based regimens against 25 on NPI regimens and showed that triglycerides and nonHDL-cholesterol were higher in PI-treated children compared with NPI-treated and untreated HIVinfected children ${ }^{10}$. However in view of the small sample size with differences in age groups, and a short study period, further studies involving larger samples and longer study period are needed to document long term effects, on clinical, immunological and adverse effect profiles.

\section{Conclusions}

- PI based regimens were effective as first line therapy in HIV infected children less than 5 years old.

- Both PI and NPI regimens were equally effective at 6 months of therapy but PI based regimens achieved significantly better growth parameters and CD4 levels.

- Elevated total cholesterol levels were observed after therapy with PI regimens.

\section{References}

1. Lodha R, Upadhyay A, Kabra SK. Antiretroviral therapy in HIV-1 infected children. Indian Pediatrics 2005; 42(8): 789-96.

PMid: 16141480

2. Patel A, Trivedi SS, Chudasama RK, Patel PK. Effect of antiretroviral therapy on clinical and immunologic disease progression in HIV positive children: Oneyear follow-up study. Journal of Family and Community Medicine 2012; 19(3): 178-83.

https://doi.org/10.4103/2230-8229.102318 PMid: 23230384 PMCid: PMC3515957

3. Shah NK, Manglani M, Shah I, Ugra D, Lodha R, TriptiPensi, et al. Guidelines for HIV care and treatment in infants and children, November 2006.

4. Antiretroviral therapy of HIV infection in infants and children: towards universal access: recommendations for a public health approach - 2010 revision.

5. Volmink J, Siegfried NL, van der Merwe L, Brocklehurst P. Antiretrovirals for reducing the risk of mother-to-child transmission of HIV infection. Cochrane Database of Systematic Reviews 2007(1): Cd003510.

https://doi.org/10.1002/14651858.cd00351 0. pub2 
6. Violari A, Lindsey JC, Hughes MD, Mujuru HA, Barlow-Mosha L, Kamthunzi $\mathrm{P}$, et al. Nevirapine versus ritonavirboosted lopinavir for HIV-infected children. New England Journal of Medicine 2012; 366(25):2380-9. https://doi.org/10.1056/NEJMoa1113249 PMid: 22716976 PMCid: PMC3443859

7. Team P-S, Babiker A, Castro nee Green $\mathrm{H}$, Compagnucci A, Fiscus S, Giaquinto $\mathrm{C}$, et al. First-line antiretroviral therapy with a protease inhibitor versus nonnucleoside reverse transcriptase inhibitor and switch at higher versus low viral load in HIV-infected children: an open-label, randomised phase $2 / 3$ trial. Lancet Infectious Diseases 2011;11(4):273-83. https://doi.org/10.1016/S14733099(10)70313-3

8. Jaspan HB, Berrisford AE, Boulle AM. Two-year outcomes of children on nonnucleoside reverse transcriptase inhibitor and protease inhibitor regimens in a South African pediatric antiretroviral program. Pediatric Infectious Disease Journal 2008; 27(11):993-8.

https://doi.org/10.1097/INF.0b013e31817a cf7b

PMid: 18818556

9. Contri PV, Berchielli EM, Tremeschin MH, Negrini BV, Salomao RG, Monteiro JP. Nutritional status and lipid profile of HIV-positive children and adolescents using antiretroviral therapy. Clinics (Sao Paulo, Brazil) 2011;66(6):997-1002. https://doi.org/10.1590/S1807-

59322011000600013

PMCid: PMC3129963
10. Charakida M, Donald AE, Green H, Storry C, Clapson M, Caslake M, et al. Early structural and functional changes of the vasculature in HIV-infected children: impact of disease and antiretroviral therapy. Circulation 2005;112(1):103-9. https://doi.org/10.1161/CIRCULATIONA HA.104.517144

PMid: 15983247

11. Contri PV, Berchielli EM, Tremeschin MH, Negrini BV, Salomao RG, Monteiro JP. Nutritional status and lipid profile of HIV-positive children and adolescents using antiretroviral therapy. Clinics (Sao Paulo, Brazil) 2011;66(6):997-1002. https://doi.org/10.1161/CIRCULATIONA HA. 104.517144

PMid: 5983247

12. Charakida M, Donald AE, Green H, Storry C, Clapson M, Caslake M, et al. Early structural and functional changes of the vasculature in HIV-infected children: impact of disease and antiretroviral therapy. Circulation 2005;112(1):103-9. https://doi.org/10.1161/CIRCULATIONA HA. 104.517144

PMid: 15983247 